\title{
$X$-ray off states and optical variability in CAL 83
}

\author{
J. Greiner ${ }^{1,2}$ and R. Di Stefano ${ }^{3,4}$ \\ 1 Astrophysical Institute Potsdam, An der Sternwarte 16, 14482 Potsdam, Germany \\ 2 Max-Planck-Institute for extraterrestrial Physics, 85741 Garching, Germany \\ 3 Harvard-Smithsonian Center for Astrophysics, Cambridge, MA 02138, USA \\ 4 Department of Physics and Astronomy, Tufts University, Medford, MA 02155, USA
}

Received 30 July 2001 / Accepted 13 March 2002

\begin{abstract}
CAL 83 was one of the first supersoft X-ray binaries (SSBs) to be discovered and is considered to be the prototype of its class. In 15 X-ray observations between 1983-1997 it was observed to have nearly constant X-ray luminosity and temperature, with the exception of one off-state in 1996 (Kahabka et al. 1996). We report on a second X-ray off-state, discovered with a Chandra ACIS-S observation in November 1999. We consider the long-term X-ray and MACHO optical light curves. We find that, during more than 7 years of monitoring by the MACHO team, CAL 83 has exhibited distinct and well-defined low, intermediate, and high optical states. Transitions between states are not accompanied by color variations. We also find that both X-ray off states were observed during optical high states and were followed by optical low states within $\sim 50$ days. We discuss possible explanations for the observed optical and X-ray variations. While photospheric adjustments might account for the variations in soft X-ray flux, optical variations can be explained only by invoking changes in the accretion disk, which is the primary source of optical radiation.
\end{abstract}

Key words. X-ray: stars - accretion, accretion disks - stars: binaries: close - stars: individual: CAL 83

\section{Introduction}

Supersoft X-ray sources are sources whose radiation is almost completely emitted in the energy band below $0.5 \mathrm{keV}$ and whose bolometric luminosity is $10^{36-38} \mathrm{erg} \mathrm{s}^{-1}$. Some SSSs are hot white dwarfs (WDs) in a post-nova phase, and some are WD or pre-WD central stars of planetary nebulae. An important subclass of SSSs are supersoft Xray binaries (SSBs). The first two SSBs to be discovered, CAL 83 and CAL 87, were observed with Einstein (Long et al. 1981), and were found to have optical properties (luminosity and periodicity) very similar to the properties of low-mass X-ray binaries (Crampton et al. 1987; Pakull et al. 1987, 1988). ROSAT observations revealed many more of these sources, and established supersoft X-ray binaries as a class (see Greiner 1996 and references therein). Several different observational facts suggest that SSBs contain white dwarfs and that many of these are burning hydrogen in a shell, with a very thin and hot atmosphere on top. The widely accepted scenario for these so-called close-binary supersoft sources (Di Stefano \& Nelson 1996), which have orbital periods of tens of hours, is based on a companion which may be slightly evolved and/or more massive than the white dwarf. These conditions allow mass

Send offprint requests to: J. Greiner,

e-mail: jcg@mpe.mpg.de transfer to occur at the high rates required to burn the accreted hydrogen in a quasi-steady manner near the white dwarf's surface (van den Heuvel et al. 1992; Rappaport et al. 1994). SSBs are known to be variable on time scales from hours to years (see Greiner 1995 for a review; or Kahabka 1996; Krautter et al. 1996; Greiner et al. 1996; Reinsch et al. 2000).

CAL 83 is the prototypical supersoft X-ray binary (SSB), and among the brightest (in terms of count rate in $\mathrm{X}$-ray detectors) at X-ray wavelengths. CAL 83 was long thought to be an X-ray persistent SSB, until Kahabka et al. (1996) discovered an X-ray off state in April 1996. Observations taken $\sim 20$ days prior to and $\sim 100$ days after the off-state, found CAL 83 to exhibit its normal X-ray brightness, thus constraining the duration of the off-state to less than 120 days (Kahabka 1998).

IUE and HST ultraviolet observations showed that the UV flux is variable by more than a factor of two (Crampton et al. 1987; Gänsicke et al. 1998). During the UV-bright state the optical emission was also brighter (Bianchi \& Pakull 1988). Crampton et al. (1987) claimed that during the UV-bright state the IUE-spectrum was steeper, thus suggesting a hotter source, but a reanalysis did not reproduce this finding (Gänsicke 2002, priv. comm.). Besides the orbital, sinusoidal variation of $1.0436 \pm 0.0044$ days (Smale et al. 1988), CAL 83 has 
also shown seemingly irregular, optical variability on a longer time scale (Pakull et al. 1985; Crampton et al. 1987; Bianchi \& Pakull 1988).

CAL 83 happened to be in a field regularly monitored by the MACHO team for more than 7 years. Based on a $\sim 150$ day portion of the MACHO observations centered around the X-ray off-state in 1996 (modified Julian Date MJD 50 200), Alcock et al. (1997) noticed an optical minimum which preceded the X-ray off-state by $\sim 10-15$ days. Alcock et al. (1997) concluded that this sequence of events is similar to the decline phase of a nova. Alternatively, Kahabka (1998) suggests that variations in the temperature of the white dwarf envelope due to expansion/contraction are the cause for the observed X-ray variability, similar to RX J0513.9-6951 (Pakull et al. 1993; Reinsch et al. 1996; Southwell et al. 1996) and AG Dra (Greiner et al. 1997).

In this paper we add two elements that lead to an alternative interpretation. First, we have used Chandra to observe a second X-ray off-state. In contrast to the 1996 $\mathrm{X}$-ray off-state, this one took place during a well-sampled optical high state and appears to have not been preceded by a recent optical low state. Second, we now have access to more than 7 years of data from the optical monitoring of the LMC by the MACHO team. We therefore combine the MACHO data with all available X-ray observations. We find that both X-ray off states occurred during optical high states, and that both were followed by an optical low state. We discuss the synthesis of all of the optical and X-ray data on CAL 83 and possible interpretations of the patterns therein.

\section{Observations and results}

\subsection{Optical observations by the MACHO team}

The MACHO Project (Alcock et al. 1995) was a microlensing survey that monitored the brightnesses of 60 million stars in the Large Magellanic Cloud, Small Magellanic Cloud, and Galactic bulge between 1992 and end of 1999. It used the $1.27 \mathrm{~m}$ Great Melbourne Telescope located at the Mount Stromlo Observatory in Australia. Two filters were used: a visual filter (4500-6300 $\AA$ ) and a red filter (6300-7600 $\AA$ ), the magnitudes of which are transformed to the standard Kron-Cousins $V$ and $R$ system, respectively. At both the red and visual foci, a mosaic of four $2048 \times 2048$ Loral CCDs are mounted.

The optical data were kindly provided by the MACHO team (courtesy A. Becker). A total of 514 observations of CAL 83 have been performed, 168 in the red and 346 in the visual filter, respectively.

Because of the rather complicated calibration issues (Alcock et al. 1999) involved in the transformation of the MACHO instrumental magnitudes into the standard Kron-Cousins $V$ and $R$ system, only those measurements can be unambiguously calibrated for which (nearly-) simultaneous red- and visual-filter observations are available. For CAL 83 this is the case for 130 observations (called two-color data in the following), while the remainder are one-color measurements. The remaining calibration zero-point uncertainty for the MACHO photometry database is estimated to be $\pm 0.10 \mathrm{mag}$ in $V$ or $R$, and $\pm 0.04 \mathrm{mag}$ in $R-V$.

The light curve of CAL 83 based on the two-color measurements is shown in Fig. 1, including the $R-V$ color. It can be seen that only marginal color changes are associated with the large intensity changes (Fig. 1). One might be tempted to "see" the color being bluer during optical high-intensity states. However, nearly all of the data points with more than 0.05 mag color variation relative to the mean value also have rather large errors (bottom panel of Figs. 1, 2). From this we conclude that the $R-V$ color is basically constant, formally $\Delta(V-R)=0.03 \pm 0.05 \mathrm{mag}$, throughout the $>1$ mag intensity changes.

In the case that the color of an object is constant, a "generic" calibration of the one-color data to a standard system can be made. As shown above, this is justified for CAL 83. We determine a mean offset between instrumental and calibrated magnitudes from the two-color data set of $24.35 \mathrm{mag}$ in the " $B$ " band, and $23.92 \mathrm{mag}$ in the " $R$ " band (Fig. 3). There could be a small $(<0.02 \mathrm{mag})$ systematic non-linear term in the $V$ band data, with the instrumental magnitude being lower (higher) at the bright (faint) end of the calibrated magnitudes (see lower panel of Fig. 3), but this is smaller than the quoted MACHO errors, so we ignore it in the following. We note, however, that if this were true, it would reduce the $\Delta(V-R)$ even further. These offsets have been applied (with the above justification) to the one-color data, and the light curve using the complete data set is shown in Fig. 4.

Before turning to the obvious variability seen in the data, it is worth mentioning that CAL 83 exhibits orbital variation of its intensity of about $\pm 0.11 \mathrm{mag}$ (Smale et al. 1988). Since the MACHO observations were not done at identical phases, these orbital variations have to be kept in mind when interpreting the light curve.

\subsection{X-ray observations}

We present the results of our recent Chandra observation and also summarize results of previous X-ray observations.

\subsubsection{Einstein}

CAL 83 was the softest and brightest source in the LMC survey which comprised 97 sources (Long et al. 1981). A comprehensive analysis of the six Einstein observations of CAL 83 is given by Brown et al. (1994). For the two long-exposure IPC observations the spectral fits result in $k T \sim 8-80 \mathrm{eV}$ depending on the adopted absorbing column. The original count rate values of Brown et al. (1994) imply a range of $0.006 \pm 0.002$ to $0.123 \pm 0.016 \mathrm{cts} / \mathrm{s}$. However, the observations were done with CAL 83 located near the edge of the field of view of the detector, with 


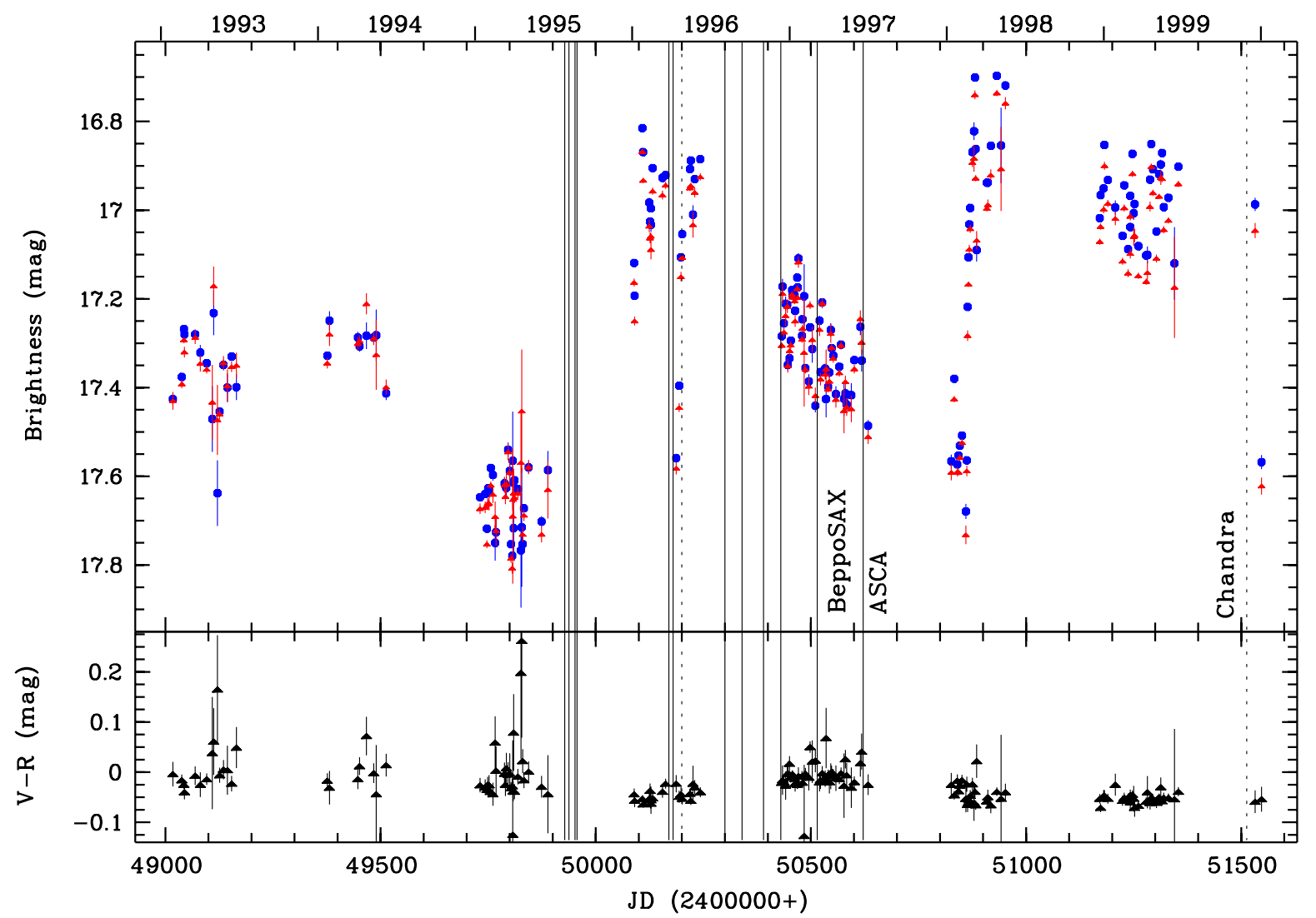

Fig. 1. MACHO light curve of CAL 83 based solely on the two-color calibrated data (see text for more details). Visual-filter ( $V$ band) observations are marked by filled circles, while red-filter $(R)$ are marked by triangles. The lower panel shows the $V-R$ color. Vertical lines denote times of X-ray observations. With the exception of observations with BeppoSAX and Chandra (individually labeled) all of these have been performed with ROSAT. The full lines indicate normal X-ray "on"-state, while dotted lines mark the two only X-ray "off"-states.

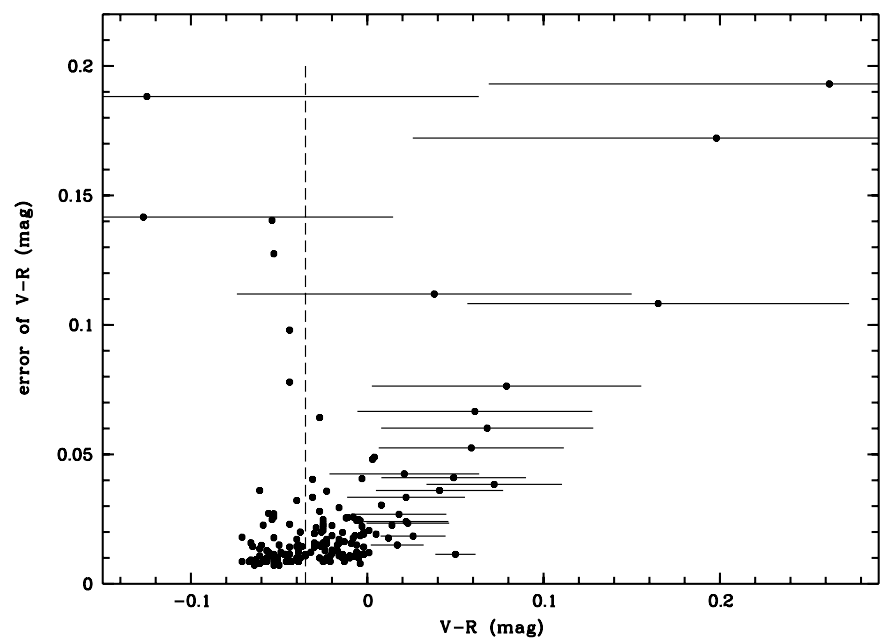

Fig. 2. Error of the color " $B$ "- " $R$ " $=V-R$ plotted over the color. Nearly all data points with more than 0.05 mag deviation from the median (dashed lines) have errors which make them compatible with the median.

off-axis angles in the $25^{\prime}-35^{\prime}$ range, thus introducing possible systematic uncertainties; furthermore, Kahabka (1998) mentions a private communication by E. Gotthelf that the implied variability could be entirely due to instrumental effects, like vignetting correction or obstruction by detector ribs. With the permission of E. Gotthelf we publish his results (which take into account these corrections) in the appendix, which indeed show CAL 83 to be constant during all IPC observations.

\subsubsection{EXOSAT}

CAL 83 was observed by EXOSAT on 1985 December 18 over a period of about $16 \mathrm{hrs}$ (Crampton et al. 1987). Most of the CMA observations were made with the Lexan filter $(0.05-2 \mathrm{keV})$, yielding a mean count rate of $0.0422 \pm$ $0.0014 \mathrm{cts} / \mathrm{s}$, but some data were also obtained with the $\mathrm{Al} / \mathrm{P}$ filter $(0.0204 \pm 0.0032 \mathrm{cts} / \mathrm{s})$. Since the Boron filter observation only provided an upper limit, not much spectral information can be deduced apart from the fact that the spectrum must be soft. Contemporaneous optical spectroscopy shows no peculiarities with respect to earlier spectroscopic data, suggesting no outburst or unusual state.

\subsubsection{ROSAT}

CAL 83 was observed by ROSAT several times, first during the verification phase in June 1990 (Greiner et al. 1991), and then during the ROSAT all-sky survey 


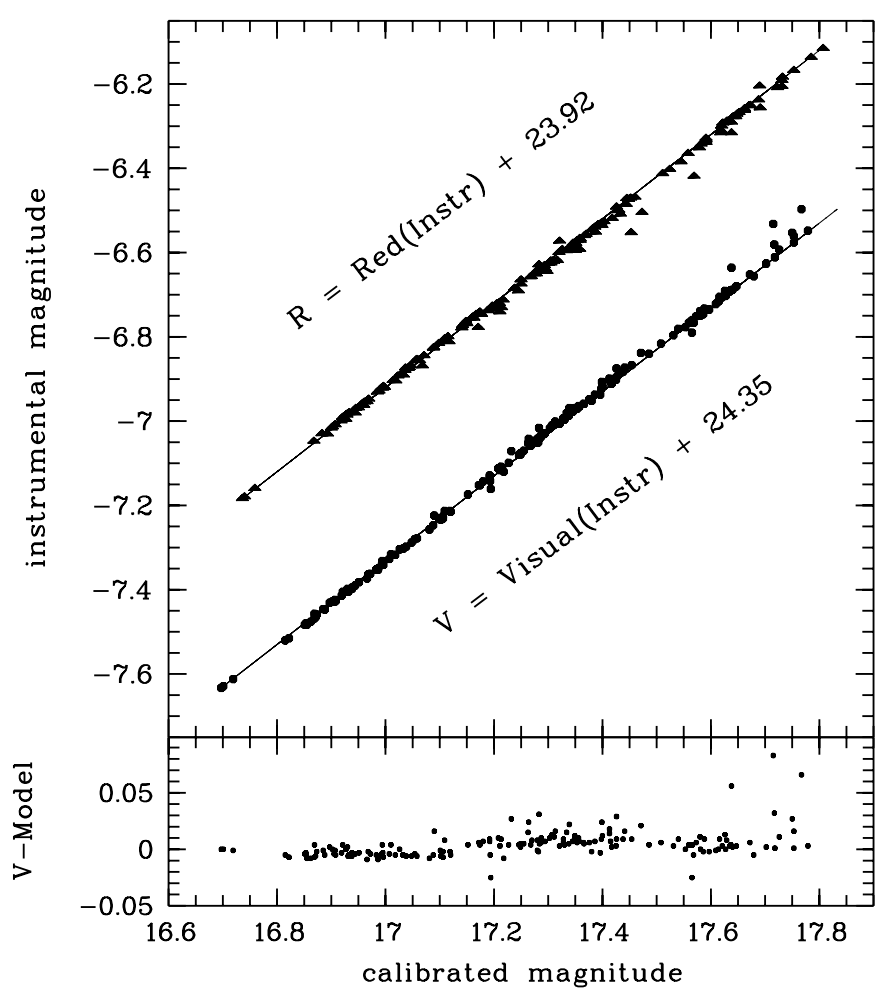

Fig. 3. Correlation of the calibrated versus instrumental magnitudes in the two-color calibrated data (top panel). With few exceptions, the linear correlations as labeled are accurate to better than $\pm 0.05 \mathrm{mag}$, though a non-linear term could be present (shown in the lower panel for the $V$ band data). We have used the linear term for a "generic" calibration of the one-color data (Fig. 4).

(1990/1991). These observations show CAL 83 at $\sim 1 \mathrm{cts} / \mathrm{s}$ in the PSPC. Spectral fitting using a blackbody model gives best-fit values of $25-45 \mathrm{eV}$ and a luminosity around $10^{38} \mathrm{erg} \mathrm{s}^{-1}$ (Greiner et al. 1991). The small variations seen in count rate and spectral shape are consistent with being due to varying response at different off-axis angles. All ROSAT pointed observations after the all-sky survey have been obtained with the HRI and were summarized and extensively discussed by Kahabka (1998). Since the MACHO data only cover the time since 1992, we just display the times of pointed ROSAT observations as given in Table 1 of Kahabka (1998). The observed count rates, except for the one observation during the April 1996 offstate, show a scatter of less than $20 \%$ around the mean of $\sim 0.2 \mathrm{HRI}$ cts/s. The X-ray off-state observation gives a $2 \sigma$ upper limit of $4.8 \times 10^{-3} \mathrm{cts} / \mathrm{s}$, which could be modeled by a temperature decrease from $39 \mathrm{eV}$ to $27 \mathrm{eV}$ (Kahabka 1998), though the exact range depends on the absolute temperatures since the count rate is a strong non-linear function with decreasing temperature.

\subsubsection{BeppoSAX}

Among several other SSBs, CAL 83 was observed with BeppoSAX on March 7/8 1997 for a total of $36 \mathrm{ksec}$ in the Low-Energy Concentrator Spectrometer (LECS).
No absorption edges have been found in this observation which could hint to a hot white dwarf atmosphere (though the low signal-to-noise ratio allows no further conclusions). Blackbody and white dwarf atmosphere models fit equally well, and result in a temperature of $30-50 \mathrm{eV}$ and a luminosity of $2-6 \times 10^{37} \mathrm{erg} \mathrm{s}^{-1}$ depending on which model is used and whether the absorbing column is fixed to the HST-derived value or left free (Parmar et al. 1998), consistent with the values derived from the ROSAT PSPC observations.

\subsubsection{ASCA}

An ASCA observation of CAL 83 was made on 21/22 June 1997, for a total of $16 \mathrm{ksec}$. The X-ray intensity was as expected for CAL 83's normal X-ray on state, and even a combined fit with ROSAT data from June 1990 was possible without any need for relative intensity adjustments (Dotani et al. 2000). As with the BeppoSAX data, the higher spectral resolution data did not require any absorption edges, and the derived effective temperature $(29 \pm 8 \mathrm{eV})$ was identical to the ROSAT value (Dotani et al. 2000).

\subsubsection{Chandra}

CAL 83 was observed within the Guaranteed-Time Observations programme with ACIS-S (PI: S. Murray) on 30 November 1999 for 2.11 ksec. Because of the better soft X-ray response, the backside-illuminated ACIS-S was chosen in the imaging observation. Since we expected about $2 \mathrm{cts} / \mathrm{s}$, the observation was performed in continuous clocking mode. CAL 83 remained undetected, the $3 \sigma$ upper limit is $0.004 \mathrm{cts} / \mathrm{s}$. Under the assumption of $k T \sim$ $40 \mathrm{eV}$ (and an absorbing column of $N_{\mathrm{H}}=6.5 \times 10^{20} \mathrm{~cm}^{-2}$; Gänsicke et al. 1998) this corresponds to a luminosity limit of $8 \times 10^{34} \mathrm{erg} \mathrm{s}^{-1}$. If, on the contrary, we assume a constant bolometric luminosity of $4 \times 10^{37} \mathrm{erg} \mathrm{s}^{-1}$, the nondetection implies either a temperature limit of $k T \lesssim 15 \mathrm{eV}$ at the canonical absorbing column, or a limit on the absorbing column of $N_{\mathrm{H}} \gtrsim 6 \times 10^{21} \mathrm{~cm}^{-2}$ at the canonical $40 \mathrm{eV}$ effective temperature.

CAL 83 was also observed with HRC-S+LETG (PI: Brinkman) for $52.3 \mathrm{ksec}$ on 29 November 1999. The nondetection in HRC-S (Murray, priv. comm.) at zeroth order implies an upper limit which is of the same order of magnitude as the ACIS-S limit (the factor 25 in exposure time is balanced by a factor 16-20 less efficiency of the grating).

\subsubsection{XMM-Newton}

On 23 April 2000, CAL 83 was observed with XMMNewton for a total of $45 \mathrm{ksec}$, aiming at a highresolution X-ray spectrum using the Reflection Grating Spectrometer (RGS) (Paerels et al. 2001). While the spectrum shows a surprising wealth of details, the source intensity is as expected from extrapolation of observed 


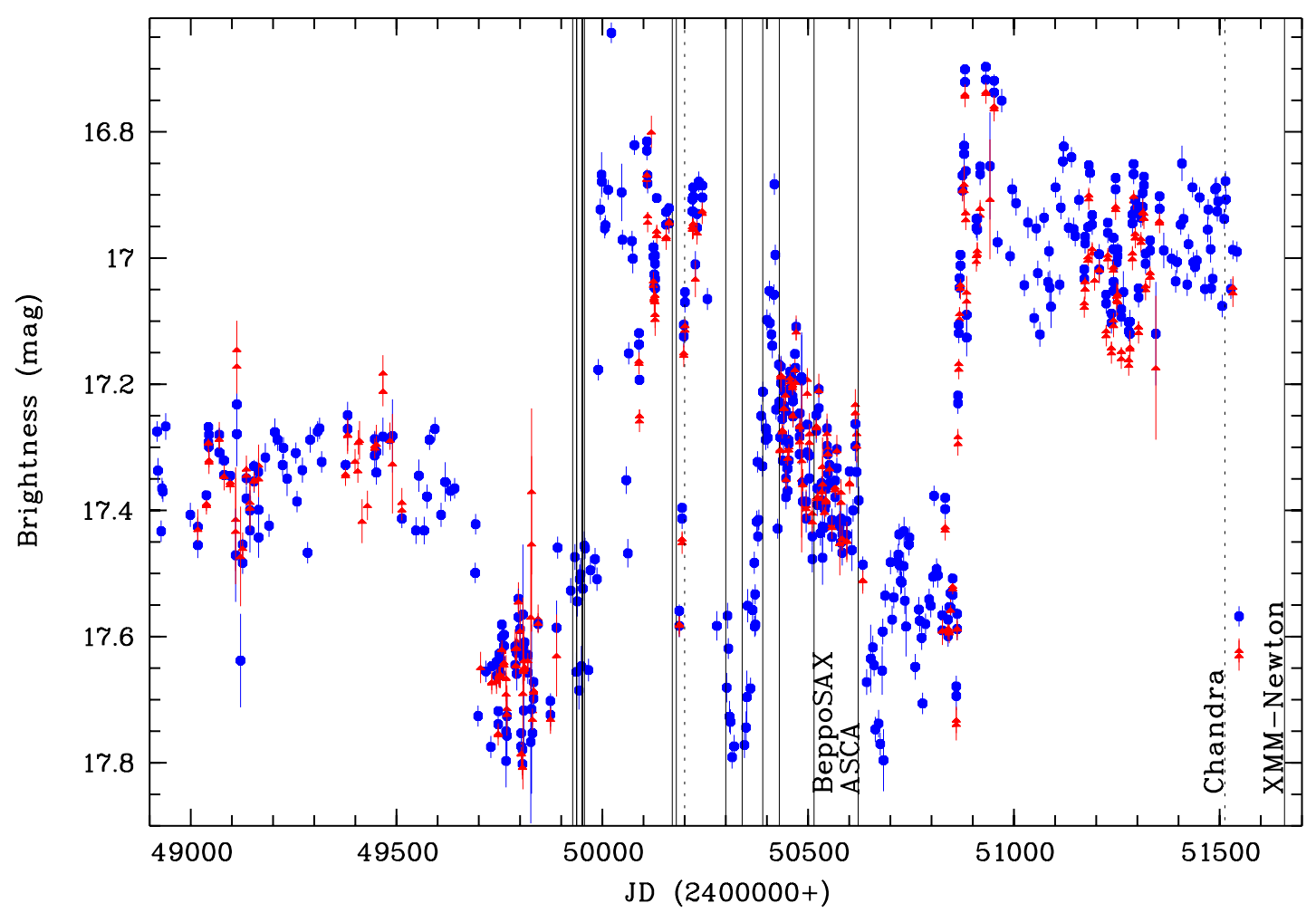

Fig. 4. MACHO light curve of CAL 83 showing also the one-color data after the "generic" correction. Times of X-ray measurements are again overplotted (see Fig. 1).

intensities with ROSAT and BeppoSAX (using PIMMS), thus indicating that by the date of this observation CAL 83 was back to its normal X-ray on-state.

\section{Patterns in the data}

\subsection{Pattern of optical variations}

During more than 7 years of MACHO observations, CAL 83 has been observed to exhibit three apparently distinct optical states. There is (1) a low state, during which the magnitude varies between roughly 17.8 and $17.5 \mathrm{mag}$; (2) an intermediate state, during which the magnitude varies between roughly 17.5 and $17.1 \mathrm{mag}$; and (3) a high state, during which the magnitude varies between roughly 17.1 and $16.7 \mathrm{mag}$. The complete MACHO light curve is displayed in Fig. 4; here we mention some of its salient features.

(a) Although there are transitions between states, the states themselves are well defined. That is, the time spent continuously within any given state is generally significantly longer than the time spent making transitions into or out of that state.

(b) In some portions of the optical light curve, variations about the mean magnitude within a state (low, intermediate, or high) seem to be comparable to the $\sim \pm 0$. 11 mag orbital variations (Smale et al. 1988), e.g. during the time periods (in modified Julian Date) $49000-$ 49600,49 700-49 900, and 50 400-50600. These variations are, of course, occurring everywhere in the light curve, but are difficult to recognize (without a period folding analysis) during times of intrinsic variability.

(c) When the system makes a transition to a higher state, it tends to "overshoot"; i.e., the luminosity soon after the transition is generally significantly $(\sim 0.2 \mathrm{mag})$ larger than the average luminosity of the system during the rest of the time in that higher state. Each of the three cases of transitions to a higher state (low to high near MJD 49 950; low to intermediate near MJD 50 400; low to high near MJD 50850 ), exhibit this behavior.

(d) During transitions, the $V-R$ color is nearly constant: $\Delta(V-R)<0.03$. We reiterate this point, because it may provide an important clue to the physics of the transitions.

(e) The MACHO data set includes a long-lived intermediate state, which likely started some time before the MACHO observations commenced and which lasted during the first $\sim 700$ days of monitoring. The MACHO data set also includes a long-lived high state, that started at roughly MJD 50875 and which lasted for just over 600 days.

(f) The MACHO data set does not include a comparably long low state; the longest lasting low states have durations of $\sim 200$ days.

(g) For a period of about 1200 days, between the long-lived intermediate state and the long-lived high state mentioned above, the system experienced a sequence of shorter-lived (200-250 day) states: low; high (punctuated by excursions to an intermediate state); low; intermediate; low. 


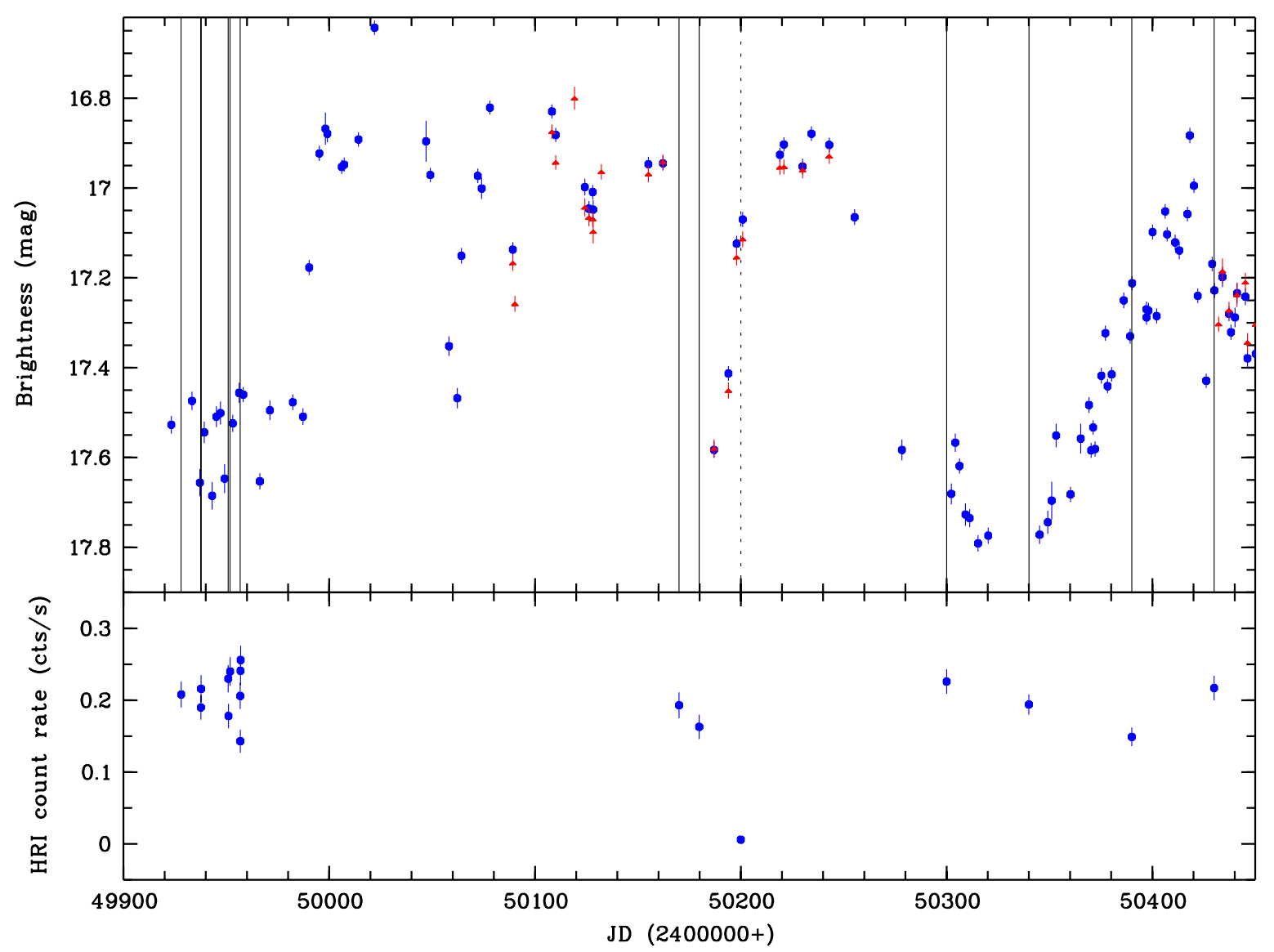

Fig. 5. Blown-up MACHO light curve of CAL 83 covering all the times of pointed ROSAT HRI observations. Times of X-ray measurements are again overplotted (see Fig. 1). The lower panel shows the ROSAT HRI count rates as published by Kahabka (1998).

(h) The intermediate state that occurred during the 1200 day interval of more frequent transitions shows a steady declining trend. Indeed, a general declining trend from high through intermediate and to low luminosity was evident from MJD 50000 to MJD 50850. This overall trend is interrupted by the optical low states that occurred immediately before and after the 1996 X-ray off state.

\subsection{X-ray variations}

Two X-ray states have been observed, an on-state, and an off-state. Though measurements with different satellites/instruments are difficult to compare, all evidence points towards a constant effective temperature and luminosity, with absolute values (depending on the applied models) of $k T \sim 30-50 \mathrm{eV}$, and $L \sim 2 \times 10^{37}$ $1 \times 10^{38} \mathrm{erg} \mathrm{s}^{-1}$. The ROSAT-detected X-ray off state represents a factor of $\gtrsim 40$ variation in count rate, while the Chandra-detected X-ray off state represents a factor of $\gtrsim 500$ variation in count rate (based on a PIMMS predicted rate for CAL 83's normal X-ray on-state using the ROSAT PSPC count rate; these predictions have been verified to be true for 6 other supersoft X-ray sources observed with Chandra ACIS-S; Greiner et al. 2002).
An X-ray observation that occurred $\sim 21$ days prior to the 1996 X-ray off-state showed the system was in its normal X-ray on-state and was apparently neither becoming less luminous nor cooler. An X-ray observation that occurred $\sim 100$ days after the 1996 X-ray off-state again found the system to be in its normal X-ray on-state. These observations place strong constraints on the decay and growth time of the X-ray flux and on changes in effective temperature, both prior to and after the off-state.

\subsection{Correlation of optical and X-ray data}

(a) It is an interesting and unfortunate fact that no Xray observations were carried out during the long-lived intermediate and high optical states covered by the MACHO data (for the preceding decades since 1980 optical observations were too sparse to allow a classification into one of the three states). The sole exception is the Chandra observation on MJD $=51513$ which occurred during the optical high state, but just before the system made a sharp transition to the optical low state.

(b) The ROSAT, ASCA, and BeppoSAX observations occurred during the period of most frequent optical-state transitions, between MJD 50025 and MJD 50625. X-ray 
on states observed during this time occurred during optical low and intermediate states.

(c) X-ray off states have occurred only during optical high states. Note that the X-ray coverage is so limited that we cannot infer that the $\mathrm{X}$-ray emission is always detectable during low and intermediate states. Nor do we know that the X-ray radiation is always undetectable during optical high states.

(d) The very last MACHO observation shows a dramatic fading which seems related to our Chandradiscovered off-state. Fortunately, several optical observations occurred for about 30 days after the Chandra observation (MJD $=51513$ ) of the X-ray off-state, with no indication of a decline. This seems to indicate a clear delay between the X-ray off and optical low states. When the optical decline occurs, however, it is relatively sudden. Indeed, the time between the last two MACHO observations is 5 days, showing that the optical fading by $0.7 \mathrm{mag}$ happened in less than 5 days (between MJD 51542 and 51547), and 29-34 days after the Chandra observation. Since the beginning (and end) of the X-ray off-state is unknown, the actual delay time between X-ray and optical low-state is longer than 29-34 days.

(e) The behaviour during the time of the April 1996 X-ray off-state (MJD around 50200 ) can be interpreted in the same way. That is, the X-ray off state is followed by optical fading around MJD = 50250 and continuing up to MJD $=50330$ ). If we adopt such an assignment, the delay of the optical fading would be about 50 days after the X-ray off-state, comparable to the interval observed in November 1999. It is attractive to apply the same scenario to explain the sequence observed for both X-ray off states. Note that the Alcock et al. (1997) scenario of optical dipping during X-ray off-states cannot apply to the Chandra-discovered off state.

\subsection{Possible patterns of correlated optical/X-ray variation}

X-ray coverage of CAL 83 has been sporadic. Even though the optical observations by the MACHO team were regular, the average time between observations was 8 days, during which both X-ray and optical fluxes were changing on comparable or possibly smaller time scales. It is therefore entirely possible that more frequent and regular observations would find behavior of a type we have not yet been able to observe.

Two patterns of correlation are consistent with the Xray and optical data taken to date.

1. X-ray off-states occur during optical high states, and $\mathrm{X}$-ray on-states during optical low and intermediate state;

2. Optical variations (e.g. decline) follow about 3050 days after an X-ray state transition (e.g. turn-off).

\section{Understanding the variations in the context of binary models}

\subsection{X-ray and optical variations in SSBs}

To determine the reasons for the X-ray and optical variations, we must consider the origin of radiation in each energy band. In the standard SSB models (van den Heuvel et al. 1992; Rappaport et al. 1994; Di Stefano \& Nelson 1996), the copious soft X-ray radiation is generated by the nuclear burning of matter near the surface of the white dwarf. In order for a WD to burn matter as it accretes, the accretion rate must be near $\sim 10^{-7} M_{\odot} \mathrm{yr}^{-1}$.

A key characteristic of SSBs, such as CAL 83 and RX J0513.9-6951 is that, while the bulk of the X-radiation is emitted by the nuclear-burning white dwarf, it is the accretion disk that is the primary source of radiation at longer wavelengths (Popham \& Di Stefano 1996). The flux and spectrum of radiation from the disk are largely determined by the reprocessing of energy from the white dwarf and not directly by the accretion process. The optical flux from the disk is significantly greater than that associated with white dwarf or its companion, the latter of which likely explains the fact that the spectra of the companions in close-binary supersoft sources have not been detected. Thus, although variations in the X-ray flux must be explained in terms of variations associated with the white dwarf, optical variations must be primarily associated with the disk.

\subsection{Variations of the $X$-ray flux}

EUV radiation and soft X-rays are generated near the white dwarf's surface, where nuclear burning is or has been occurring. X-ray variability can be due to changes in the accretion rate, burning rate, or photospheric radius. At rates larger than the burning rate, matter which accretes but fails to burn may form a swollen envelope, or can be ejected in the form of winds.

\subsubsection{Does the WD central engine turn on and off?}

It is reasonable to ask whether the apparent cessation of $\mathrm{X}$-ray radiation at two times in the light curve of CAL 83 is likely to be a signal that nuclear burning had ceased, and that the central source was no longer emitting $\mathrm{X}$ rays. This is unlikely, because it takes time for the white dwarf to cool. Those novae which have been observed as SSSs have taken months or even years to cool enough that they are no longer observed as X-ray sources, like GQ Mus (Ögelman et al. 1993); V1974 Cyg (Krautter et al. 1996), Nova LMC 1995 (Orio \& Greiner 1999). We do not know the mass of the white dwarf likely to be the central source in CAL 83. However, the low X-ray luminosity (Gänsicke et al. 1998) as well as the white dwarf model fits to BeppoSAX data (Parmar et al. 1998) suggest that the mass is not high $\left(\sim 0.9-1.0 M_{\odot}\right)$. A variety of computations (e.g. Prialnik \& Kovetz 1995; Kato 1997, 1999) 
suggest that cooling (i.e. turn-off) times can be as short as 6-20 days only if the mass of the white dwarf is near the Chandrasekhar limit; otherwise the cooling time (for the emission to be shifted out of the X-ray regime) is in excess of several months. It therefore seems highly unlikely that a cessation of nuclear burning occurred around MJD 50200 in 1996, leading to the X-ray off-state found by Kahabka et al. (1996). It is possible that nuclear burning ceased much earlier, but in this case, the X-ray detections prior to MJD 50200 should have shown some indication of evolution toward a cool state. Since there is no evidence of such evolution, we conclude that the off-state in 1996 was unlikely to be related to a complete cessation of nuclear burning and subsequent cooling of the white dwarf.

This does not mean there might not have been a modulation of the bolometric luminosity during an interval of time around the off state, perhaps due to a modulation of the accretion rate, or perhaps due to a change in the short-term average energy release associated with nuclear burning. But it does mean that a hot white dwarf, with luminosity roughly comparable to its value at earlier and later times, was still present in CAL 83, even during the X-ray off-state in 1996. Occam's razor would therefore suggest that the WD was also hot during the Chandra-discovered off-state.

The question then becomes, if the hot white dwarf was there, why did we not detect X-rays during these 2 observations? There are two possibilities.

1. The white dwarf's energy emission may be shifted toward longer wavelengths. This could happen if, e.g., the radius of the photosphere increases;

2. X-rays emitted by the white dwarf could interact with matter in the vicinity of the system; the energy thus absorbed would eventually be re-emitted at other wavelengths.

\subsubsection{Photospheric adjustments}

Expansion of the photosphere can shift the peak of the spectrum toward longer wavelengths. Because the X-ray emission we detect from SSBs constitutes only the highenergy tail of the emitted spectrum, even modest shifts of the peak to lower energies can shift the tail so that $\mathrm{X}$-ray instruments are no longer able to detect the source. Modulations of the photospheric radius can be caused by adjustments of the accretion rate, or by changes associated with nuclear burning, even at relatively steady accretion rate. Photospheric adjustments seem to be the most direct way to achieve the observed X-ray off-states, in cases in which the bolometric luminosities do not apparently suffer a large decline and an X-ray on-state re-occurs soon thereafter.

Indeed this scenario has shown to work for the novae GQ Mus (Ögelman et al. 1993) and V1974 Cyg (Krautter et al. 1996), and has been applied to similar sources like the SSB RX J0513.9-6951 (Reinsch et al. 1996;
Southwell et al. 1996) or the symbiotic system AG Dra during outburst (Greiner et al. 1997). RX J0513.9-6951 is the most prominent example, varying quasi-periodically at both X-ray and optical wavelengths. Because the variations repeat at relatively short time intervals, the variations of RX J0513.9-6951 are much better studied than those of CAL 83. In particular, RX J0513.9-6951 goes through optical low-states lasting $\sim 30-40$ days and repeating quasi-regularly at 150-180 days intervals. The decrease in the optical flux of $\Delta V \sim 1 \mathrm{mag}$ is accompanied by a reddening of $\Delta(B-V) \sim 0.1-0.2 \mathrm{mag}$ and $\Delta(V-R) \sim 0.1 \mathrm{mag}$ (Reinsch et al. 1996). Soft X-ray emission is only observed during the short optical lowstates, while during the long optical high states no X-ray emission is detected. There have been two different suggestions to explain this behaviour:

1. If during optical high states the accreting white dwarf is slightly (by about a factor of 3) expanded and cool enough to not emit X-rays, then a temporarily slightly reduced mass-transfer rate would cause the photospheric radius to shrink. As a consequence, optical emission from the white dwarf would be reduced, and later on the white dwarf would get hot enough $(k T \gtrsim 20 \mathrm{eV})$ to be detectable in X-rays (Pakull et al. 1993; Southwell et al. 1996);

2. Taking into account the fact that in RX J0513.9-6951 most of the light from the accretion disk is reprocessed emission, Reinsch et al. (1996) proposed that it is not the white dwarf itself which is producing the varying optical emission, but the changes of the white dwarf induce variations of the irradiation of the accretion disk which in turn are the cause for the observed optical variations.

Interestingly enough, the physical parameters of RX J0513.9-6951 (X-ray flux, temperature, orbital period) are similar to those of CAL 83. Consequently, both models have been invoked to explain CAL 83's variations (Kahabka 1998). Alternatively, Alcock et al. (1997) suggested a cessation of nuclear burning on the white dwarf in CAL 83, leading to a behaviour similar to the decline phase of a nova. We have argued above that a cessation of nuclear burning in CAL 83 is very unlikely. We will argue below that the Kahabka model with its additional assumption of a particularly shaped mass transfer modulation for CAL 83 does not explain all the presently available data. Instead, a more complicated variant of model (2) seems to be required:

1. The variability pattern is much more complicated in CAL 83 than in RX J0513.9-6951, i.e. not just a relatively fast switching between two brightness levels;

2. CAL 83 shows no color changes (Figs. 1, 2). Both photospheric adjustments as well as a varying wind (absorption) would predict substantial color changes ( $\gtrsim 0.1 \mathrm{mag}$ ), and the binary inclination is comparable in both systems. Though CAL 83 and RX J0513.96951 have slightly different disk sizes as implied by 
their different orbital periods (1.04 days vs. 0.76 days) and luminosities, the relative changes (in $\dot{M}$, color etc.) should be similar;

3. Sophisticated modelling of the UV/X-ray observations of CAL 83 has resulted in a bolometric luminosity of $(0.7-2) \times 10^{37} \mathrm{erg} \mathrm{s}^{-1}$ (Gänsicke et al. 1998). The inferred mean luminosity averaged over the last $\sim 10^{5}$ years as derived from the [O III] and $\mathrm{H} \alpha$ lines of the ionization nebula is $(1-7) \times 10^{37} \mathrm{erg} \mathrm{s}^{-1}$ (Remillard et al. 1995), implying that X-ray off states should represent only a small fraction over the last $\sim 10^{5}$ years. However, relating X-ray off states to optical high states (above pattern 1) would be statistically inconsistent with such a small fraction, since CAL 83 was in an optical high state for $\sim 30 \%$ over the last 10 years;

4. The overshooting after transitions from optical low or intermediate state to high state is difficult to explain by photospheric radius changes.

We also note that even the expanded white dwarf cannot outshine the accretion disk, unless it grows to $5 \times 10^{11} \mathrm{~cm}$, larger than the binary separation of $3.7 \times 10^{11} \mathrm{~cm}$.

\subsubsection{Absorption}

Absorption due to material above the burning surface could also have the effect of shifting the effective radius. If, for example, (1) more matter is accreting per second than can, on average, be burned during one second, and (2) not all of this matter can be instantaneously ejected, it does not take long for the "extra" mass to interfere with the detection of X-rays. Suppose that the ejected winds are preferentially emitted in a cone (jet) oriented perpendicular to the accretion disk as deduced from the broad He and $\mathrm{H} \alpha$ wings (Crampton et al. 1987; Cowley et al. 1998). During an interval in which a substantial fraction of material incident from the donor is neither burned nor ejected as winds, $\sim 10^{19} \mathrm{~g} \mathrm{~s}^{-1}$ may accumulate over an area smaller than $\left(10^{9}\right)^{2} \mathrm{~cm}^{2}$. X-ray radiation could be quenched on relatively short time scales, as unburned matter begins to seep upward through what had been the wind cone. The seeping matter can affect both our view of the disk and also the radiation incident on the disk from the central star. Note that, in the case of CAL 83, the blocking of the disk from our view is unlikely to be the only cause of optical variation. Although this model is consistent with the lack of color change, it would not lead to a spike in the optical emission when the disk again becomes visible. The existence of the overshooting apparent in the light curve tells us that some real adjustment of the disk is likely to be taking place as it moves from one equilibrium state to another.

\subsection{Variations of the optical flux}

Variations of the optical flux are most likely to be associated with changes in the accretion disk. The accretion disks surrounding the white dwarf in SSBs are bright, generally more than an order of magnitude brighter than the accretion luminosity (viscous heating). This is because, in contrast to the situation for canonical cataclysmic variables or neutron star binaries, nuclear burning of accreted material releases ( $\sim 20$ times) more energy, than the potential energy lost and dissipated through accretion. Reprocessing of this radiation by the disk is of particular importance in CBSSs (e.g. Popham \& Di Stefano 1996; Matsumoto \& Fukue 1998; Fukue \& Hachiya 1999; Suleimanov et al. 1999). If $\sim 20 \%$ of the energy emitted by the white dwarf is reprocessed by the disk, the disk luminosity will be $10^{35}-10^{37}$ erg $\mathrm{s}^{-1}$. Farther from the white dwarf, the disk is cooler due to the less pronounced heating, becoming the dominant source of UV and optical light. The height of the outer disk flares (Popham \& Di Stefano 1996; Meyer-Hofmeister et al. 1997). The geometry of the disk emerges from first-principles calculations (Popham \& Di Stefano 1996) and, for the eclipsing CBSS CAL 87, can also be derived phenomenologically (Schandl et al. 1997).

If roughly $20 \%$ of the energy released due to nuclear burning is reprocessed by the disk, and if roughly one half of the accretion energy is dissipated in and radiated from the disk, then accretion energy accounts for only $\sim 10 \%$ of the energy emitted by the disk. Changes in the accretion rate produce changes in the disk luminosity on the order of a few (perhaps as much as 10) percent. These changes are likely to occur prior to any changes in X-ray flux due to a changing rate of fuel delivery by the disk.

A cessation of nuclear burning could eventually (after cooling) cause the disk to become 2 orders of magnitude less luminous, even if accretion continued at a comparable rate.

Unless they constitute just a small fraction of an upward or downward trend, luminosity changes by a factor of $\sim 2$ are most likely due to changes directly related to the reprocessing of radiation from the white dwarf. Reprocessing changes are of two types. First, reprocessing that occurs as radiation directly from the central star hits and interacts with the disk, is naturally influenced by changes in the spectrum or flux of radiation from the central star. Second, heating from the central star through the disk will also be affected. The flow of heat is influenced by the size and temperature of the photosphere as well as by the instantaneous luminosity of the white dwarf. This heating is communicated from the white dwarf through matter in the disk. The disk response can therefore lag the X-ray response.

\subsection{Correlations between X-ray and optical flux}

The primary cause for the variations is either a modulation of the rate at which the companion donates matter, or a modulation of the rate of nuclear burning (though the observed time scale of state changes in CAL 83 is very short for a supposed white dwarf mass of $\left.\sim 1 M_{\odot}\right)$. In each case, the bolometric luminosity will change, even as 
the changes affect different parts of the spectrum differently. Prompt anticorrelations between X-ray and optical flux suggest photospheric changes that shift the flux distribution from the X-ray range towards longer wavelengths and immediately influence the disk by affecting the spectrum of light incident on it from the white dwarf. Delayed correlations or anticorrelations suggest that matter flowing to the white dwarf may be responding to different local conditions as disturbances due to changing accretion rates and differences in heating from the white dwarf propagate through the disk.

\section{Summary and prospects}

CAL 83 is one of the flagship sources of the class of supersoft X-ray binaries. It may therefore seem strange that CAL 83 was discovered to have an X-ray off state 16 years after it was first observed by Einstein. In common with many other X-ray sources, CAL 83 has been observed only sporadically. It may therefore not be so surprising that we have little information about its X-ray variations. Furthermore, although RXTE has conducted regular allsky monitoring, its detectors are not sensitive to the soft radiation emitted by CAL 83 .

Two alternative interpretations of the pattern of Xray and optical variations are consistent with the data on CAL 83: (1) X-ray off-states occur during optical high states, and X-ray on-states during optical low and intermediate state, or (2) optical variations follow about 30 50 days after an X-ray state transition.

We have shown above that neither the simple picture of photospheric radius changes nor pure absorption effects can be the primary cause for these observed patterns in CAL 83. Instead, changes in the photospheric radius lead to changes in the irradiation and heating of the disk, perhaps at a time when the accretion rate is also (or has been) changing. It is this suite of changes, accompanying any photospheric change, that modulate the optical luminosity. Optical variations can therefore have a more complex relationship to photospheric expansion or contraction.
One of the things we have learned, however, is that optical monitoring programs, like those carried out to search for evidence of microlensing, can complement the information gleaned from X-ray observations. For RX J0513.96951, for example, a combination of early optical observations specifically designed to learn more about the X-ray source (Reinsch et al. 1996) and subsequent monitoring by the MACHO team (Alcock et al. 1997), allow us to now use the optical data to predict the X-ray state. This also seems to be true of the VY Scl stars, in which a proposed anticorrelation between optical and X-ray fluxes allowed us to discover that at least one member of this class is a supersoft X-ray binary (Greiner et al. 1999). For CAL 83, we clearly need a better record of its X-ray light curve, coupled with optical monitoring, to better understand the relationship between its optical and X-ray flux.

Based on the pattern observed to date and the previous discussion it is clear that simultaneous and frequent multiwavelength observations of CAL 83 are needed to test physical models for the source and its variability. Such observations can provide important clues to the physics of this interesting system and the SSB class to which it belongs.

Acknowledgements. We are highly indebted to A. Becker for making the calibrated MACHO data available to us. We also want to thank Andrea Dupree for enlightening discussions. Thanks also to the CfA data processing group for extensive data validation checks of the Chandra observation of CAL 83 in Nov. 1999. We appreciate the generosity of Eric Gotthelf for making available his re-analysis of the CAL Einstein data for publication herein (see Appendix). We are grateful to the referee, P. Kahabka, for the careful reading and detailed comments which improved the presentation of the data. This work was supported in part by NSF under INT-9815655 and by NASA under NAG5-10705.

\section{Appendix}

Below are the results of a re-analysis of the Einstein IPC data by Eric Gotthelf (Columbia University, USA) from

Table 1. Einstein IPC observations of CAL 83 (by E.V. Gotthelf).

\begin{tabular}{ccccccccc}
\hline Seq\# & $\begin{array}{c}\text { ObsTime } \\
(\mathrm{JD})\end{array}$ & $\begin{array}{c}\text { Pointing }(\mathrm{B} 1950) \\
\text { RA }\end{array}$ & $\begin{array}{c}\text { Roll } \\
\text { Dec }\end{array}$ & $\begin{array}{c}\text { Livetime } \\
(\mathrm{deg})\end{array}$ & $\begin{array}{c}\text { Src Cts }^{a} \\
(\mathrm{~s})\end{array}$ & $\begin{array}{c}\text { Offaxis } \\
(2-10 \mathrm{ch})\end{array}$ & $\begin{array}{c}\text { Rate }_{(\text {error })^{b}} \\
\left(r=3^{\prime}\right)\end{array}$ \\
\hline 2417 & 2443974.09981 & $54343-681159$ & 66 & 573 & 61 & 11 & $0.090(0.018)$ \\
2418 & 2443972.99426 & $54904-681159$ & 68 & 1690 & 16 & 31 & $0.060(0.05)^{c}$ \\
2430 & 2443972.78994 & $54743-684159$ & 68 & 899 & 80 & 28 & $0.120(0.02)$ \\
6301 & 2444280.04540 & $54312-685819$ & 124 & 23361 & 1419 & 35 & $0.108(0.003)$ \\
7109 & 2444279.08445 & $54329-675059$ & 125 & 13841 & 1049 & 32 & $0.120(0.004)$ \\
\hline
\end{tabular}

\footnotetext{
Notes:

${ }^{a}$ Background subtracted source counts in a $3^{\prime}$ radius aperture centered on CAL 83.

${ }^{b}$ Summed exposure corrected count rate in a $3^{\prime}$ radius aperture (error $\sqrt{(S+B) /(S-B)}$ ), where $S$ and $B$ are the source and background count rate, respectively.

${ }^{c}$ This number is unreliable. The source was partially obscured by an IPC rib.
} 
1996, which have been mentioned as "priv. comm." in Kahakba (1998) but were never published. These results correct the wrong count rates of Brown et al. (1994). As can be seen from the table below, there does not seem to be any significant source variability $(<10 \%)$. Notice that the observation closest to the optical axis has the lowest count rate. In contrast, one would expect a systematic increase in the count rate if the difference was due to instrumental effects.

\section{References}

Alcock, C., et al. 1995, PRL, 74, 2867

Alcock, C., Allsman, R. A., Alves, D., et al. 1997, MNRAS, 286,483

Alcock, C., Allsman, R. A., Alves, D., et al. 1999, PASP, 111, 1539

Bianchi, L., \& Pakull, M. W. 1988, in A Decade of UV Astronomy with IUE, ed. E. J. Rolfe, ESA-SP 281, 145

Brown, T., Cordova, F., Ciardullo, R., Thompson, R., \& Bond, H. 1994, ApJ, 422, 118

Cowley, A. P., Schmidtke, P. C., Crampton, D., \& Hutchings, J. B. 1998, ApJ, 504, 854

Crampton, D., Cowley, A. P., Hutchings, J. B., et al. 1987, ApJ, 321, 745

Di Stefano, R., \& Nelson, L. A. 1996, in Supersoft X-ray Sources, ed. J. Greiner, Lecture Notes Phys. 472 (Springer), 3

Dotani, T., Asai, K., Ebisawa, K., \& Greiner, J. 2000, COSPAR, Nagoya, 12-19 July 1998, Adv. Space Res., $25(3 / 4), 375$

Fukue, J., \& Hachiya, M. 1999, PASJ, 51, 185

Gänsicke, B. T., van Teeseling, A., Beuermann, K., \& de Martino, D. 1998, A\&A, 333, 163

Greiner, J. 1995, Abano-Padova Conf. on Cataclysmic variables, ed. A. Bianchini, M. Della Valle, \& M. Orio, ASSL, 205,443

Greiner, J. 1996, Supersoft X-ray Sources, Lect. Notes in Physics 472 (Springer)

Greiner, J., Schwarz, R., Hasinger, G., \& Orio, M. 1996, A\&A, 312,88

Greiner, J., Bickert, K. F., Luthardt, R., et al. 1997, A\&A, 322,576

Greiner, J., Tovmassian, G. H., Di Stefano, R., et al. 1999, A\&A, 343, 183

Greiner, J., Di Stefano, R., Murray, S., et al. 2002, in preparation
Kahabka, P. 1996, A\&A, 306, 795

Kahabka, P., Haberl, F., \& Parmar, A. N. 1996, IAU Circ., 6467

Kahabka, P. 1998, A\&A, 331, 328

Kato, M. 1997, ApJS, 113, 121

Kato, M. 1999, PASJ, 51, 525

Krautter, J., Ögelman, H., Starrfield, S., Wichman, R., \& Pfeffermann, E. 1996, ApJ, 456, 788

Long, K. S., Helfand, D. J., \& Grabelsky, D. A. 1981, ApJ, 248,925

Matsumoto, K., \& Fukue, J. 1998, PASJ, 50, 89

Meyer-Hofmeister, E., Schandl, S., \& Meyer, F. 1997, A\&A, 321,245

Ögelman, H., Orio, M., Krautter, J., \& Starrfield, S. 1993, Nature, 361, 331

Orio, M., \& Greiner, J. 1999, A\&A, 344, L13

Paerels, F., Rasmussen, A. P., Hartmann, H. W., et al. 2001, A\&A, 365, L308

Pakull, M. W., Beuermann, K., Angebault, L. P., \& Bianchi, L. 1987, Ap\&SS, 131, 689

Pakull, M. W., Beuermann, K., van der Klis, M., \& van Paradijs, J. 1988, A\&A, 203, L27

Pakull, M. W., Motch, C., Bianchi, L., et al. 1993, A\&A, 278, L39

Parmar, A. N., Kahabka, P., Hartmann, H. W., Heise, J., \& Taylor, B. G. 1998, A\&A, 332, 199

Popham, R., \& Di Stefano, R. 1996, in Supersoft X-ray Sources, ed. J. Greiner, Lecture Notes Phys. 472 (Springer), 65

Prialnik, D., \& Kovetz, A. 1995, ApJ, 445, 789

Rappaport, S., Di Stefano, R., \& Smith, J. D. 1994, ApJ, 426, 692

Reinsch, K., van Teeseling, A., Beuermann, K., \& Abbott, T. M. C. 1996, A\&A, 309, L11

Reinsch, K., van Teeseling, A., King, A. R., \& Beuermann, K. 2000, A\&A, 354, L37

Remillard, R. A., Rappaport, S., \& Macri, L. M. 1995, ApJ, 439, 646

Schandl, S., Meyer-Hofmeister, E., \& Meyer, F. 1997, A\&A, 318, 73

Smale, A. P., Corbet, R. H., Charles, P. A., et al. 1988, MNRAS, 233, 51

Southwell, K. A., Livio, M., Charles, P. A., O'Donoghue, D., \& Sutherland, W. J. 1996, ApJ, 470, 1065

Suleimanov, V., Meyer, F., \& Meyer-Hofmeister, E. 1999, A\&A, 350, 63

van den Heuvel, E. P. J., Bhattacharya, D., Nomoto, K., \& Rappaport, S. A. 1992, A\&A 262, 97 